\title{
Synthèse des études radioécologiques annuelles menées dans l'environnement des centrales électronucléaires françaises depuis 1991
}

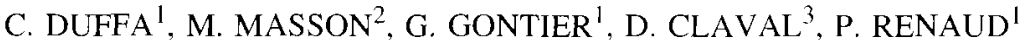

(Manuscrit reçu le 17 octobre 2003, accepté le 14 février 2004)

RÉSUMÉ Depuis 1991, l'IRSN réalise un suivi radioécologique annuel de l'environnement des installations électronucléaires françaises. Plus de 5000 échantillons, prélevés dans le milieu terrestre et aquatique autour des $\mathbf{2 0}$ sites étudiés, ont ainsi été analysés par spectrométrie gamma à bas niveau. Cette synthèse permet d'établir un bilan de 11 ans d'études, en présentant les objectifs et méthodes mises en ceuvre, et en énonçant les principales observations et conclusions qui peuvent ressortir de l'analyse des résultats de mesures obtenus. Il apparaît que les rejets atmosphériques chroniques de radionucléides émetteurs gamma effectués par les CNPE ne conduisent le plus souvent pas à des apports décelables dans l'environnement des sites. Ces observations ont conduit l'IRSN à réduire le nombre d'analyses portant sur des échantillons du milieu terrestre à partir de 2000 . Les rejets liquides sont, quant à eux, responsables d'un marquage de l'environnement aquatique, mis en évidence par la détection de faibles activités de ${ }^{60} \mathrm{Co},{ }^{58} \mathrm{Co},{ }^{110 \mathrm{~m}} \mathrm{Ag}$ on ${ }^{54} \mathrm{Mn}$, et des différences significatives des valeurs des rapports d'activités ${ }^{137} \mathrm{Cs}^{134} \mathrm{Cs}$ mesurés en aval des installations. Les apports de radioactivité gamma artificielle en Manche sont également décelables dans les échantillons marins prélevés à proximité des CNPE. Toutefois, ce marquage peut être difficile à mettre en évidence du fait de la présence des radionucléides artificiels rejetés par les installations de COGEMA-La Hague. Au-delà des informations importantes que ces études apportent quant au marquage de l'environnement par les rejets des CNPE, les résultats acquis depuis 1991 constituent une source importante de données qui nous informent sur le devenir et la répartition des radionucléides dans l'environnement.

ABSTRACT Review of annual radioecological studies carried out since 1991 in the French nuclear power plants environment.

Since 1991, the IRSN carries out annual radioecological studies in the environment of the French Nuclear Power Plants. More than 5,000 samples, collected in terrestrial and aquatic ecosystems around the 20 studied plants, have been analysed by low-level gamma spectrometry. This paper presents the main goals and methods for such studies, and the lessons learnt from 11 years results. The French NPP routine atmospheric releases do not lead to detectable radioactive inputs into their surroundings. For this reason, IRSN decided to reduce the number of analysis concerning terrestrial samples since 2000 . On the other hand, NPP liquid discharges

\footnotetext{
1 IRSN, DEl/SESLRE/LERCM. Centre d'etudes de Cadarache. B.P. 3, 13115 Saint-Paul-Lez-Durance, France

2 IRSN, DEI/SECRE/LRC, B.P. 10.50130 Cherbourg-Octeville, France.

ONECTRA, Centre d'études de Cadarache, bâtiment 153. B.P. 3, 1,3115 Saint-Paul-Lez-Durance. France.
} 
into rivers are responsible for the presence of low ${ }^{60} \mathrm{Co},{ }^{58} \mathrm{Co},{ }^{110 \mathrm{~m}} \mathrm{Ag}$ and ${ }^{54} \mathrm{Mn}$ activities and significant difference in ${ }^{137} \mathrm{Cs} /{ }^{134} \mathrm{Cs}$ activity ratios measured in aquatic compartments. Radioactive discharges of artificial gamma emitters are also detectable in the Channel marine environment around NPP. Nevertheless, this influence is often concealed by radionuclides released by COGEMA-La Hague nuclear reprocessing plant. Beyond important evaluations concerning the presence of artificial radionuclides in NPP's environment, studies conducted since 1991 give us an important database that can be used for a better knowledge of transfers and distribution of radioactivity through the environment.

\section{Introduction}

La production d'électricité en France est assurée aujourd'hui à $76 \%$ par l'énergie d'origine nucléaire, via 58 réacteurs à eau sous pression (REP) exploités par EDF (Électricité de France), et répartis sur 19 sites différents (Fig. 1). Le surgénérateur de Creys-Malville, qui a fonctionné jusqu'en 1998 est actuellement en déconstruction. Il est implanté sur un vingtième site mentionné sur la figure 1.

Malgré les dispositifs de rétention et de filtration des effluents, des produits radioactifs sont rejetés sous contrôle par voies liquide et atmosphérique lors du fonctionnement normal de ces $20 \mathrm{CNPE}$ (Centres nucléaires de production d'électricité). L'étude de l'environnement des sites nucléaires permet de rendre compte de l'état radioécologique des différents milieux, et nous informe quant au devenir des radionucléides in situ.

Pour chacun des 20 sites précités, de telles études ont été menées, à la demande de l'exploitant, à différentes périodes du fonctionnement des installations. L'IRSN a ainsi réalisé une étude initiale (ou « point zéro ») pour chaque site, visant à dresser un premier état radioécologique de l'environnement avant démarrage du premier réacteur. À partir de ce bilan initial, tous les 10 ans, un bilan décennal permet d'étudier l'évolution des activités des radionucléides émetteurs gamma, alpha et bêta présents dans les différents compartiments de l'environnement. De plus, depuis 1991, l'évolution de la radioactivité gamma autour de chaque CNPE est suivie annuellement (IRSN, 1992 à 2002). Les mesures réalisées dans le cadre de ces études ne constituent pas les campagnes de contrôle réglementaire des demandes d'autorisation de rejets et de prélèvements d'eau (DARPE) auquel sont soumis les exploitants. Il s'agit de suivre l'incidence du fonctionnement normal de chaque installation sur la radioactivité de son environnement, d'améliorer les connaissances des phénomènes de transferts et d'accumulation des radionucléides dans les différents milieux, et de disposer de résultats exploitables lors d'évaluation de risques radiologiques (Foulquier et Bretheau, 1998). 


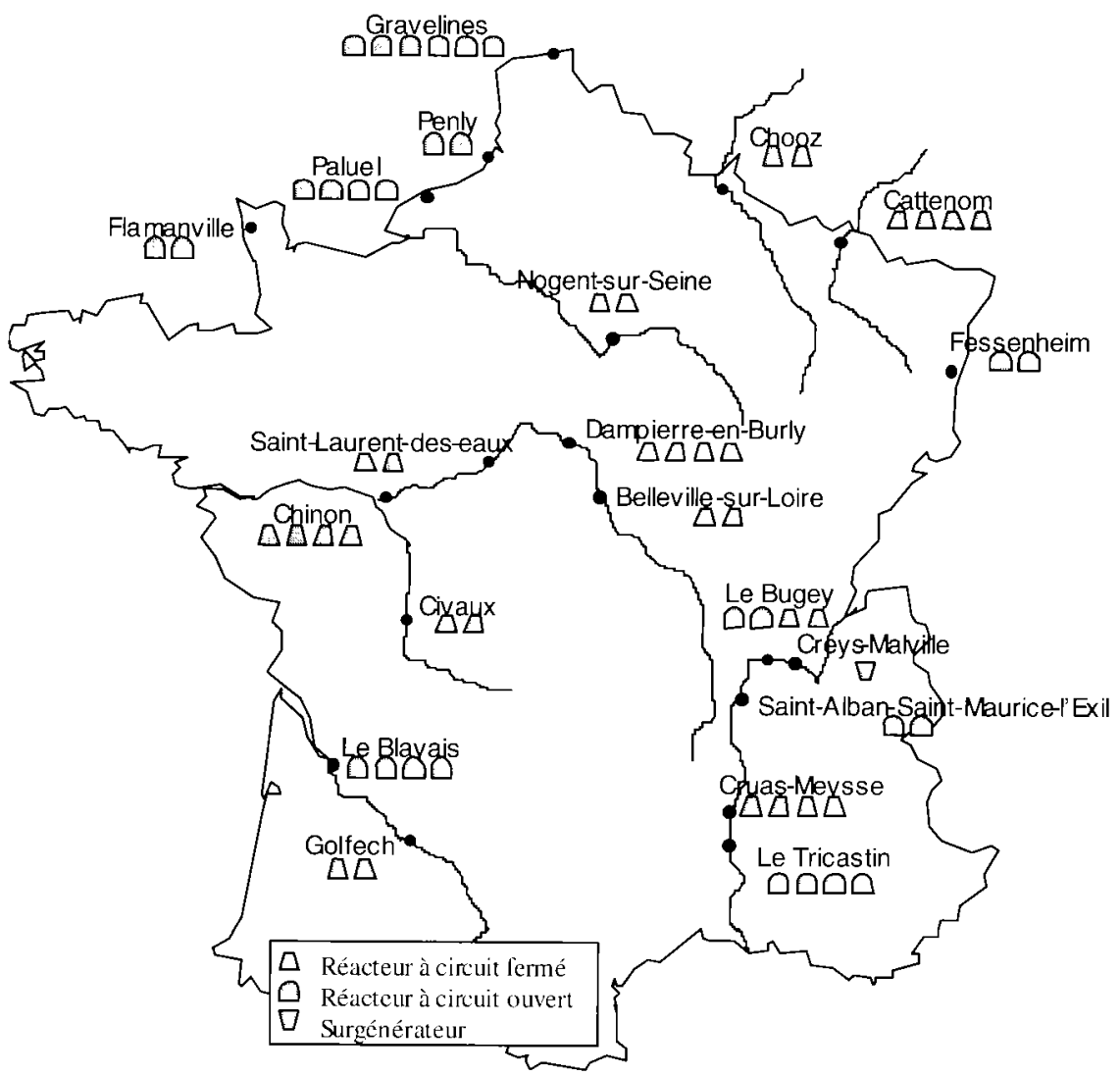

Figure 1 - Situation géographique des 20 CNPE français faisant l'objet d'un suivi radioécologique annuel.

Localization of the 20 French NPP where annual radioecological study is carried out.

L'interprétation des résultats de mesures de radioactivité consiste, en particulier, en une identification et une quantification des apports pour chacun des radionucléides significativement mesurés.

L'objectif de cette publication est de présenter une synthèse de 11 ans d'étude de la radioactivité gamma autour des CNPE français. Ce bilan permettra, après un rappel des différentes sources de radioactivité gamma pouvant conduire à des apports mesurables dans l'environnement, de revenir sur l'évolution de la stratégie d'étude, puis d'énoncer les principaux résultats et enseignements acquis à partir de l'analyse des résultats obtenus entre 1991 et 2001. 


\section{Origines des radionucléides émetteurs gamma mesurés dans l'environnement}

Tout échantillon prélevé dans l'environnement est porteur de radioactivité. Ainsi, outre les radionucléides d'origine naturelle tellurique ou cosmogénique, plusieurs radionucléides d'origine artificielle sont omniprésents dans l'environnement. Localement, les rejets effectués par les installations nucléaires peuvent conduire à un enrichissement de certains compartiments de l'environnement en radionucléides artificiels.

\subsection{Radionucléides naturels}

Les mesures réalisées par spectrométrie gamma sur des échantillons de l'environnement permettent de mettre en évidence la présence de plusieurs radionucléides d'origine naturelle. Ce sont, d'une part, des radionucléides primordiaux, constitutifs de la croûte terrestre $\left({ }^{40} \mathrm{~K},{ }^{238} \mathrm{U},{ }^{232} \mathrm{Th}\right.$ et $\left.{ }^{235} \mathrm{U}\right)$ et leurs descendants. D'autre part, ce sont les radionucléides cosmogéniques, formés dans l'atmosphère ou à l'interface air-sol lors de réactions nucléaires induites par les particules constituant le rayonnement cosmique. C'est notamment le cas du ${ }^{7} \mathrm{Be}$, produit par capture neutronique ou spallation dans l'atmosphère. Il est détecté lors des mesures gamma sur des matrices environnementales ayant intégré des apports atmosphériques.

\subsection{Radionucléides artificiels omniprésents en France}

Plusieurs radionucléides artificiels $\left({ }^{137} \mathrm{Cs},{ }^{134} \mathrm{Cs}\right.$ et ${ }^{241} \mathrm{Am}$ en particulier) sont mesurables par spectrométrie gamma dans les échantillons de l'environnement. Les dépôts globaux de ces radionucléides, liés aux retombées atmosphériques des essais aériens d'armes nucléaires pratiqués entre 1945 et 1980 , et au passage en mai 1986 du panache radioactif consécutif à l'accident de Tchemobyl, ne se sont pas répartis de manière homogène sur le territoire français. En effet, différents travaux ont déjà permis de montrer les corrélations existantes entres les hauteurs de précipitations et les activités déposées pour ces deux sources (Mitchell et al., 1990 ; Hölgye et Fildas, 1995 ; Renaud et al., 2003). L'utilisation des rapports d'activités entre différents isotopes présents est un outil précieux de caractérisation de ces termes sources. Ainsi, les isotopes $137\left(\mathrm{~T}_{1 / 2}=30,2\right.$ ans) et, dans une moindre mesure, $134\left(\mathrm{~T}_{1 / 2}=2,1\right.$ ans) du césium ont été mesurés régulièrement hors de l'influence des installations nucléaires, entre 1991 et 2001 , période que couvre cette synthèse. Le rapport d'activités ${ }^{137} \mathrm{Cs} /{ }^{134} \mathrm{Cs}$, calculé lorsque le ${ }^{134} \mathrm{Cs}$ est décelable, permet de «tracer » 1'origine de la contamination. Ce rapport a été estimé à 2,0 0,2 en 1986 dans les dépôts qui se sont produits en France suite à 


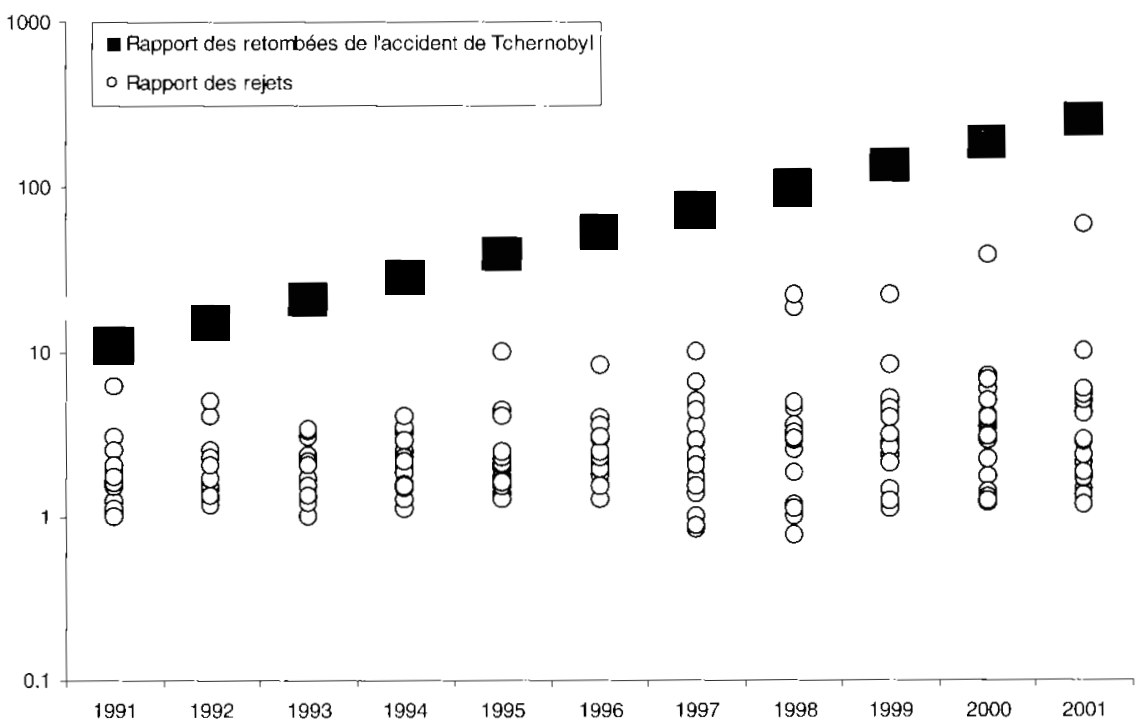

Figure 2 - Rapports d'activités ${ }^{137} \mathrm{Cs} /{ }^{134} \mathrm{Cs}$ des rejets liquides des CNPE et rapports caractéristiques des retombees de l'accident de Tchernobyl.

${ }^{137} \mathrm{Cs} /{ }^{134} \mathrm{Cs}$ activity ratios in NPP liquid discharges and Chernobyl fallout characteristic ratios.

l'accident de Tchernobyl (UNSCEAR, 1988 ; Renaud et al., 1999). À cause de la différence des périodes radioactives des deux isotopes, cette valeur augmente dans le temps. De ce fait, entre mai 1991 et mai 2001 , le rapport d'activités ${ }^{137} \mathrm{Cs} /{ }^{134} \mathrm{Cs}$ caractéristique de cette source est passé de $10 \pm 1$ à $256 \pm 22$ (Fig. 2). Par ailleurs, seul l'isotope 137 du césium était présent dans les retombées des essais atmosphériques. Ainsi, tout échantillon prélevé en France, hors de l'influence d'un autre apport significatif de Cs, est caractérisé par un rapport d'activités ${ }^{137} \mathrm{Cs} /{ }^{134} \mathrm{Cs}$ égal ou supérieur à la valeur caractéristique des retombées de Tchernobyl.

\subsection{Les rejets des CNPE}

Les radionucléides émetteurs gamma présents dans les effluents radioactifs des CNPE peuvent être des produits d'activation neutronique ou des produits de fission. Dans les REP (réacteurs à eau sous pression), ${ }^{54} \mathrm{Mn},{ }^{58} \mathrm{Co},{ }^{60} \mathrm{Co},{ }^{110 \mathrm{~m}} \mathrm{Ag}$ et ${ }^{124} \mathrm{Sb}$ sont par exemple générés par l'activation de matériaux constitutifs des structures des installations ; ${ }^{134} \mathrm{Cs},{ }^{137} \mathrm{Cs}$ et ${ }^{131} \mathrm{I}$ sont des produits de fission (EDF, 1995). 


\section{DUFFA et al.}
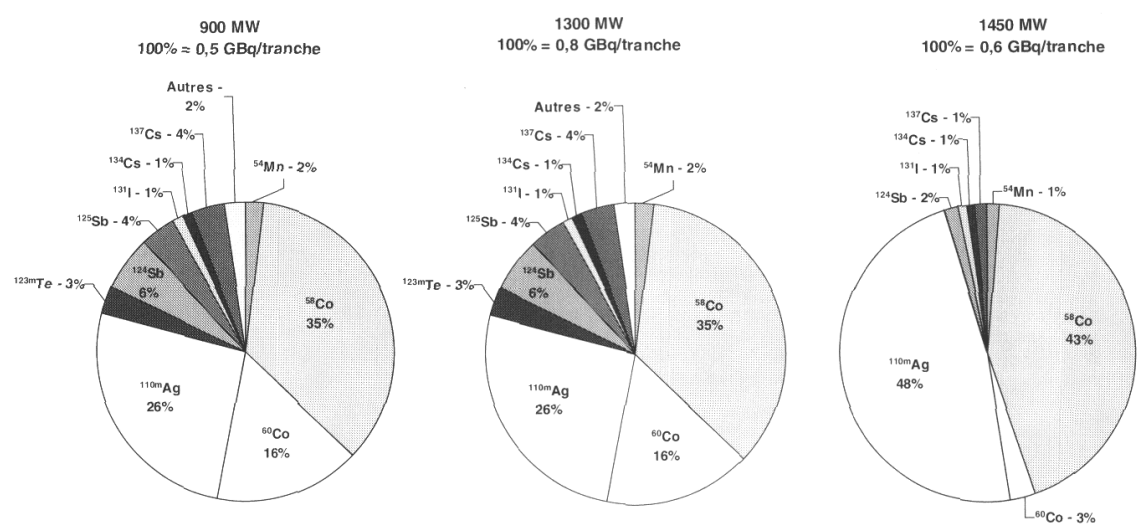

Figure 3 - Composition moyenne des effluents liquides des CNPE en 2001, émetteurs gamma (EDF, 2001).

Mean composition of NPP liquid discharges in 2001, gamma emitters (EDF, 2001).

\subsubsection{Rejets liquides}

Près de $80 \%$ de l'activité des émetteurs gamma rejetée dans les effluents liquides est due aux radionucléides ${ }^{58} \mathrm{Co},{ }^{60} \mathrm{Co},{ }^{110 \mathrm{~m}} \mathrm{Ag}$ et ${ }^{124} \mathrm{Sb}$ (Fig. 3). Les rejets liquides de césium ne constituent pas une part prépondérante en terme d'activité. Cependant, depuis 1991 , les rapports d'activités ${ }^{137} \mathrm{Cs} /{ }^{134} \mathrm{Cs}$ moyens caractéristiques des effluents des CNPE français sont inférieurs au rapport caractéristique des dépôts de Tchernobyl (Fig. 2). Ils constituent alors un outil util d'analyse des résultats de mesures des échantillons de l'environnement prélevés à proximité des installations nucléaires.

\subsubsection{Rejets atmosphériques}

Les rejets atmosphériques sont composés de gaz rares, tritium, ${ }^{14} \mathrm{C}$, halogènes et aérosols. Les activités des radionucléides émetteurs gamma mesurées au niveau des rejets sont, la plupart du temps, inférieures aux limites de détection. Les rejets déclarés par l'exploitant sont calculés sur la base de ces valeurs limites et correspondent en moyenne à $1 \%$ des limites annuelles autorisées.

\subsection{Autres sources potentielles à considérer}

Outre les origines précédemment citées, il est possible que d'autres sources soient responsables de la présence de radionucléides émetteurs gamma artificiels dans l'environnement des CNPE. On mentionnera ici les rejets en mer des usines de 
retraitement du combustible de COGEMA-La Hague et de BNFL-Sellafield (Grande Bretagne), conduisant à un marquage de l'environnement marin pouvant être décelé à proximité des CNPE de Flamanville, Paluel, Penly et Gravelines. De même, en amont du site de Fessenheim, les centrales nucléaires suisses sont susceptibles d'être à l'origine d'un marquage des eaux du Rhin.

De plus, les utilisations médicales, industrielles ou à des fins de recherche, de radionucléides artificiels $\left({ }^{131} \mathrm{I},{ }^{60} \mathrm{Co}\right.$, ou ${ }^{125} \mathrm{Sb}$ par exemple), peuvent conduire ponctuellement à des rejets dans les cours d'eau et en mer.

\section{Méthode d'étude}

\subsection{Stratégie d'échantillonnage}

Le choix d'un plan d'échantillonnage est conditionné par l'objectif recherché, le niveau de variabilité de la population cible, le nombre de mesures techniquement et parfois financièrement réalisables, et par les contraintes liées au terrain. Dans le cas des études annuelles des CNPE, il est nécessaire de choisir des lieux de prélèvement et des matrices susceptibles de nous informer sur le marquage de l'environnement par les rejets liquides ou atmosphériques des installations. C'est le rôle de l'analyse de bioindicateurs tels que les bryophytes ou les lichens. De plus, les résultats obtenus doivent nous permettre de quantifier localement les activités des principaux radionucléides émetteurs gamma dans les milieux d'accumulation (sols, sédiments) et dans les produits végétaux ou animaux consommés localement. Enfin, afin de rendre les études des 20 sites français cohérentes et d'établir si besoin des comparaisons, les stratégies d'échantillonnage sont établies de manière la plus proche possible d'un site à l'autre.

La figure 4 présente l'évolution du nombre d'analyses gamma effectuées lors du suivi radioécologique annuel depuis 1991, ainsi que les évènements qui ont marqué cette évolution. À partir de 2000 , le nombre de mesures effectuées annuellement pour chaque site a été réduit. Cette diminution concerne en particulier le milieu terrestre, peu soumis aux rejets des installations. La stratégie visant à mesurer systématiquement le même type d'échantillon, prélevé dans différentes zones plus ou moins soumises à l'influence des rejets atmosphériques chroniques des sites (zones définies en fonction des roses des vents locales), a été modifiée. Dès lors, seule une partie de ces échantillons est analysée (échantillons prélevés dans les zones les plus exposées aux apports atmosphériques), les échantillons terrestres prélevés en zones non influencées étant conservés dans une banque d'échantillons afin d'en disposer au cas où les analyses faites montreraient un marquage lié aux rejets de l'installation. D'autres révisions de la stratégie 


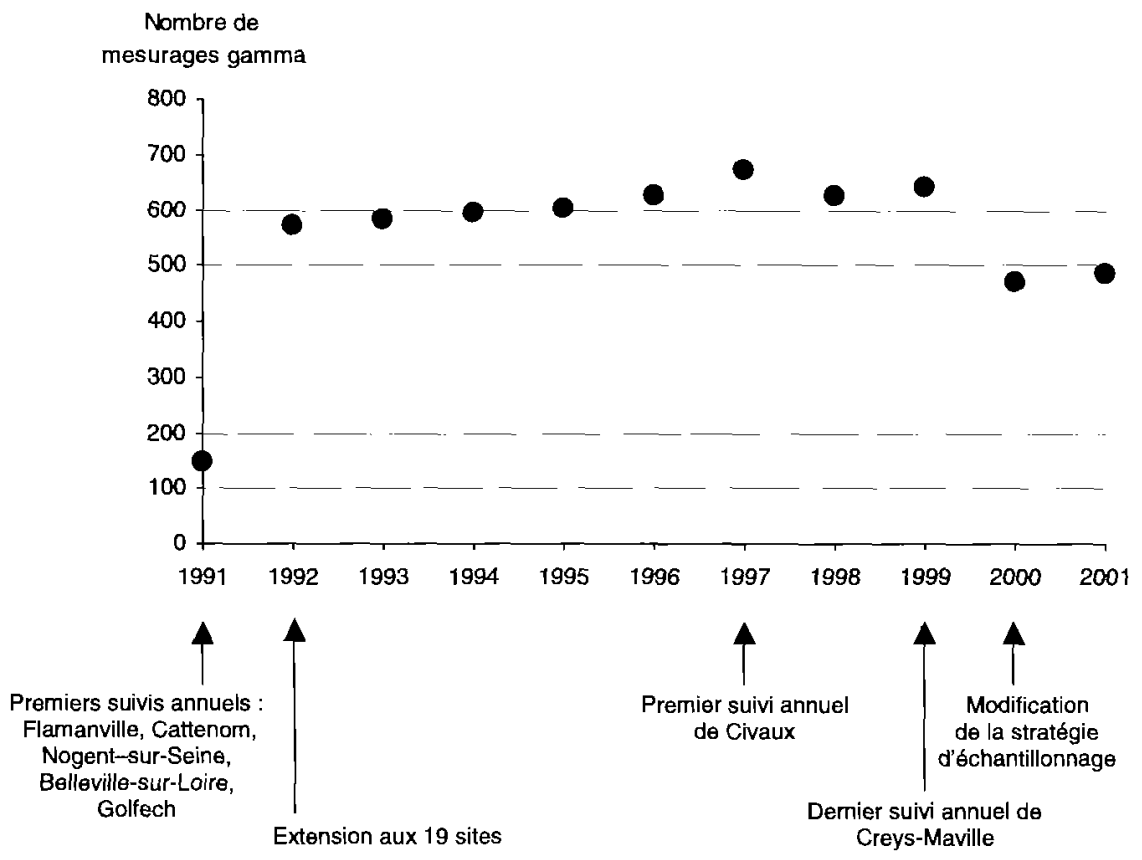

Figure 4-Évolution du nombre de prélèvements et de mesurages gamma effectués dans le cadre des études radioécologiques annuelles des CNPE entre 1991 et 2001.

Number of samplings and measurements carried out for annual NPP radioecological studies between 1991 and 2001.

d'échantillonnage sont intervenues: les analyses portant sur un échantillon d'herbe de prairie et le lait associé ont été systématisées à tous les sites, afin de disposer de séries de données cohérentes sur les produits de consommation ; dans le milieu aquatique, le nombre de mesures portant sur les poissons, présentant une part importante de résultats inférieurs aux limites de détection, a été réduit.

Sur l'ensemble de la période 1991-2001, plus de 5000 échantillons ont été prélevés, répartis à parts égales entre milieux terrestre et aquatique (continental et marin). Sur chacun d'entre eux, une ou deux mesures par spectrométrie gamma, selon le fractionnement effectué, ont été réalisées. Le tableau I synthétise le nombre de mesures effectuées avant et après le changement de stratégie mis en application en 2000, par type de matrices. On notera que ce bilan inclut des mesures gamma cffectuées en plus pour certains sites les années correspondant aux études décennales. 
SYNTHÈSE DES ÉTUDES RADIOÉCOLOGIQUES ANNUELLES

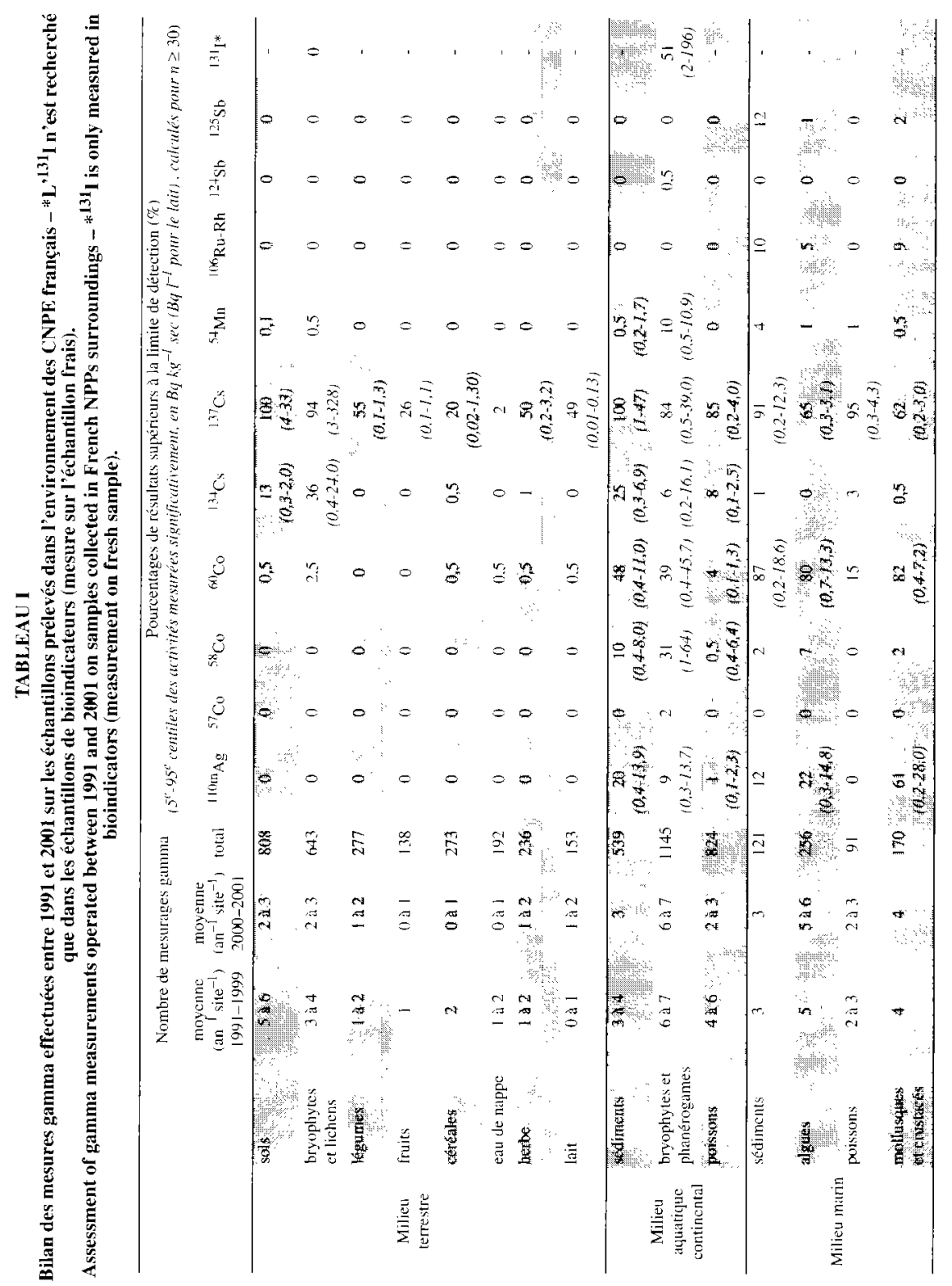


Les matrices minérales (sols et sédiments) et végétales ont été les plus étudiées (Tab. I). Les espèces bioindicatrices (bryophytes terrestres et aquatiques, lichens, phanérogames, algues) rendent compte de l'état radioécologique de l'environnement. Elles permettent de déceler des pollutions discrètes difficile à mettre en évidence par l'analyse directe de l'eau, des sols ou de l'air (définition selon Ramade, 1998). Les eaux de nappes, les matrices animales et les produits dérivés sont analysés afin d'augmenter les connaissances sur la chaîne trophique, et évidemment pour leur importance en matière de radioprotection. Dans cette catégorie, les mollusques et crustacés tiennent une place particulière car ce sont aussi de bons intégrateurs de radionucléides.

\subsection{Mesures}

Depuis 1991, près de 6000 mesures par spectrométrie gamma ont été réalisées pour l'acquisition de données informant sur l'état radioécologique de l'environnement des CNPE français. Des mesures physico-chimiques complémentaires (notamment des mesures de granulométrie et teneurs en matière organique) ont été effectuées sur certaines matrices afin d'aider à l'interprétation des données. Dans le cadre de cette synthèse, seuls les résultats de mesurages gamma ont été repris.

Avant d'effectuer la mesure elle-même, les échantillons sont traités afin d'homogénéiser la matrice et concentrer les isotopes porteurs de l'activité que l'on cherche à mesurer dans un minimum de volume (lyophilisation, séchage ou calcination des échantillons). Dans certain cas, et notamment pour la mesure de ${ }^{1}{ }^{131} \mathrm{I}$, élément volatil, les échantillons peuvent être mesuré à l'état frais. La recherche et la quantification des activités des principaux radionucléides émetteurs $\gamma$ sont réalisées par spectrométrie directe (Bouisset et Calmet, 1997). Les résultats rendus sont accompagnés de l'incertitude statistique, calculée pour un intervalle de confiance de $95 \%$.

\section{Résultats}

L'ensemble des résultats de mesures acquis, les caractéristiques des échantillons et des traitements réalisés avant les mesurages (séchage, broyage, etc.) ont été enregistrés dans la base de données SYLVESTRE ${ }^{4}$. Cela permet, a posteriori, d'extraire et d'analyser spécifiquement des séries de données choisies et d'assurer la traçabilité de toutes les opérations associées sous assurance de la qualité.

\footnotetext{
${ }^{4}$ SYLVESTRE est une base de données interne IRSN, dédiée à la gestion des échantillons et au stockage des résultats de mesures obtenus dans le cadre des études radioécologiques menées dans l'environnement.
} 


\section{TABLEAU II}

Activités en radionucléides naturels mesurées autour des $\mathrm{CNPE}\left(\mathrm{Bq} \mathrm{kg}^{-1} \mathrm{sec}, \mathrm{Bq} \mathrm{I}^{-1}\right.$ pour le lait $)$. Les résultats sont donnés sous la forme : médiane $\left(5^{\mathrm{e}}-95^{\mathrm{e}}\right.$ centile) des résultats significatifs (pour $\mathbf{n} \geq 30$ ).

Natural radionuclides activities measured around $\mathrm{NPP}\left(\mathrm{Bq} \mathrm{kg}^{-1} \mathrm{dry}, \mathrm{Bq}^{-1}\right.$ for milk). Results are given as: median (5th-95th percentile) of significant results (for $n \geq 30$ ).

\begin{tabular}{|c|c|c|c|c|c|c|}
\hline & & $\begin{array}{l}\text { PF/PS* } \\
\text { moyens }\end{array}$ & ${ }^{40} \mathrm{~K}$ & ${ }^{232} \mathrm{Th}$ & ${ }^{238} \mathrm{U}$ & ${ }^{210} \mathrm{~Pb}$ \\
\hline \multirow{7}{*}{$\begin{array}{l}\text { Milieu } \\
\text { terrestre }\end{array}$} & sols cultives & 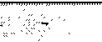 & $501(223-1151)$ & $37(12-77)$ & $40(19-82)$ & $50(2580): 6(215)$ \\
\hline & bryophytes & - & $255(140-430)$ & $10(3-26)$ & $12(3-33)$ & $384(142-938) 406(140-859)$ \\
\hline & $\begin{array}{l}\text { feguntes } \\
\text { feulles }\end{array}$ & 192 & $203(1300-3264)$ & $4\left(\frac{1+j}{1}\right)^{*}$ & ates : & $13(3,32) \% 76(19248)$ \\
\hline & fruits & 7,4 & $372(227-935)$ & - & - & $-\quad 7(3-19)$ \\
\hline & cerétes & $1 * 3$ & $166(102-940)$ & $0,4(0,20,8)$ & Whan & , स(1-21) \\
\hline & $\begin{array}{l}\text { herhes el } \\
\text { pâtures }\end{array}$ & 4,1 & $950(400-16.35)$ & $2(1-10)$ & - & $149(31-445)$ \\
\hline & 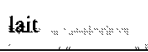 & - & $55(43-72)$ & 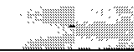 & mity & 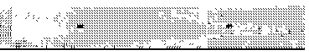 \\
\hline \multirow{4}{*}{$\begin{array}{c}\text { Milieu } \\
\text { aquatique } \\
\text { continental }\end{array}$} & sédiments & - & $660(203-1142)$ & $45(17-97)$ & $41(16-85)$ & $71(22-170)$ \\
\hline & Eryeptytes & min & $341(169-525)$ & $31(8-104)$ & $24(7-67)$ & $50(1699), 164(58-386)$ \\
\hline & phanérogames & - & $916(457-1650)$ & $14(3-50)$ & $13(5-36)$ & $41(12-136)$ \\
\hline & Peissons & 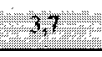 & $383(191-594)$ & $2(0,5,4,5)$ & F & $3(1-9)$ \\
\hline \multirow{4}{*}{$\begin{array}{l}\text { Milieu } \\
\text { marin }\end{array}$} & sédiments & - & $230(47-580)$ & $9(2-22)$ & $21(5-70)$ & $28(10-100) \quad 33(3-1.33)$ \\
\hline & atgues andina & (2) & $127(367-1795)$ & $4(2-10)$ & $10(422)$ & $12(3+9), 14(9-49)$ \\
\hline & $\begin{array}{l}\text { mollusques } \\
\text { (chair) }\end{array}$ & 4,9 & $343(199-569)$ & - & $7(2-12)$ & $17(5-36)$ \\
\hline & POissons: & 40 & $456(242-797)$ & 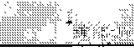 & Hy & Warn \\
\hline
\end{tabular}

"Rapports « poids frais / poids sec ».

Il est important de remarquer que les activités présentées dans cet article sont données par unité de masse sèche. Cela permet de s'affranchir des problèmes liés en particulier aux variations des teneurs en eau pour les échantillons collectés en milieu aquatique. Cependant, les rapports moyens de masses entre états frais et sec sont indiqués dans le cas des produits pour lesquelles des comparaisons pourraient être faites avec d'autres données de la littérature (Tab. II).

\subsection{Radioactivité d'origine naturelle}

Le tableau II présente les gammes de valeurs représentatives des activités massiques des radionucléides naturels les plus souvent mesurés pour chaque type de matrice. Signalons que ces activités sont parfois quantifiées de manière indirecte, par l'intermédiaire d'un radionucléide produit par filiation, et à l'équilibre séculaire. C'est notamment le cas de l, ${ }^{238} \mathrm{U}$ (on mesure le ${ }^{234} \mathrm{Th}$ ) et du ${ }^{232} \mathrm{Th}$ (on mesure l, ${ }^{228} \mathrm{Ac}$ ). 
Les valeurs données dans le tableau II sont établies à partir des résultats dits « significatifs », c'est-à-dire supérieurs aux limites de détection. Dès lors que le nombre de résultats significatifs disponibles pour un type d'échantillon est inférieur à 30, les valeurs statistiques descriptives ne sont pas présentées. Lorsque aucune valeur n'est donnée ici (par exemple pour $1{ }^{, 238} \mathrm{U}$ dans les céréales), cela signifie que les activités massiques présentes dans le compartiment considéré sont faibles, le plus souvent inférieures aux limites de détection du radionucléide considéré.

Les teneurs en ${ }^{40} \mathrm{~K},{ }^{238} \mathrm{U}$ et ${ }^{232} \mathrm{Th}$ mesurées dans les sols cultivés sont cohérentes avec les valeurs publiées par l'UNSCEAR (2000) : respectivement $400 \mathrm{~Bq} \mathrm{~kg}{ }^{-1}$, $35 \mathrm{~Bq} \mathrm{~kg}^{-1}$ et $30 \mathrm{~Bq} \mathrm{~kg}^{-1}$ (valeurs médianes pour 42 pays considérés).

Le ${ }^{40} \mathrm{~K}$ est toujours détecté dans les échantillons de l'environnement étudiés. Il constitue la principale source de radioactivité dans tous les compartiments analysés, excepté dans le cas particulier des bryophytes terrestres, pour lesquelles ${ }^{7} \mathrm{Be}$ et ${ }^{210} \mathrm{~Pb}$ sont des contributeurs majeurs. D'origine tellurique, le ${ }^{40} \mathrm{~K}$ est, comme le ${ }^{232}$ Th et $\mathrm{l}^{238} \mathrm{U}$, essentiellement concentré dans les matrices minérales (sols et sédiments). Les activités en ${ }^{40} \mathrm{~K},{ }^{232} \mathrm{Th}$ et ${ }^{238} \mathrm{U}$ des produits végétaux doivent essentiellement s'expliquer par des phénomènes connus de transferts du sol vers les plantes.

Les bryophytes terrestres sont de bons intégrateurs pour le ${ }^{7} \mathrm{Be}$ et le ${ }^{210} \mathrm{~Pb}$, radionucléides apportés en totalité $\left({ }^{7} \mathrm{Be}\right)$, ou pour partie $\left({ }^{210} \mathrm{~Pb}\right)$, par voie atmosphérique. Dans le milieu aquatique continental, la répartition de ces deux éléments entre les matrices diffère, rendant compte de comportements physicochimiques distincts. En effet, alors que pour le milieu terrestre, les activités massiques mesurées sont essentiellement le reflet des dépôts atmosphériques directs, la contamination des différents compartiments du milieu aquatique se fait indirectement, par les apports en phase dissoute ou particulaire des radionucléides déposés sur les sols du bassin versant dont le cours d'eau est tributaire. De même, dans le milieu marin, les échantillons de sédiments, végétaux ou animaux analysés ne sont pas directement exposés aux contaminations atmosphériques, mais indirectement, soit suite à des transferts dans la colonne d'eau après un apport atmosphérique, soit par des apports terrigènes.

\subsection{Radioactivité d'origine artificielle}

Parmi les résultats obtenus, on peut retenir ici 11 radionucléides émetteurs gamma artificiels pour lesquels il est possible de présenter un bilan suite à onze années d'acquisition : ${ }^{110 \mathrm{~m}} \mathrm{Ag},{ }^{57} \mathrm{Co},{ }^{58} \mathrm{Co},{ }^{60} \mathrm{Co},{ }^{134} \mathrm{Cs},{ }^{137} \mathrm{Cs}\left({ }^{137 \mathrm{~m}} \mathrm{Ba}\right),{ }^{54} \mathrm{Mn},{ }^{106} \mathrm{Ru}$ 
$\left({ }^{106} \mathrm{Rh}\right),{ }^{124} \mathrm{Sb},{ }^{125} \mathrm{Sb},{ }^{131} \mathrm{I}$. Le tableau I présente les pourcentages de résultats supérieurs aux limites de détection des techniques de mesures à très bas niveau. Il est intéressant de noter que seul le ${ }^{137} \mathrm{Cs}$ est mesurable dans toutes les matrices environnementales. La fréquence de détection d'autres radionucléides artificiels, et en particulier des isotopes du cobalt, de l'argent et de l'antimoine, traceurs des rejets des CNPE, varie en fonction des milieux et des matrices analysés.

Pour chacun des trois milieux étudiés, les gammes d'activités mesurées par matrice entre 1991 et 2001 sont fournies lorsqu'il existe plus de 30 résultats supérieurs aux limites de détection (Tab. I). Les valeurs médianes ne sont pas calculées car elles sont jugées peu représentatives à cause des hétérogénéités spatiales (entre tous les sites étudiés) et temporelles (entre 1991 et 2001) des activités de la plupart des radionucléides quantifiés.

\subsection{Milieu terrestre}

À la lecture du tableau I, il apparaît clairement que les radionucléides artificiels dont la présence témoigne d'un marquage de l'environnement pas les rejets des installations nucléaires, n'ont été détectés significativement que dans quelques rares cas dans le milieu terrestre. Le ${ }^{60} \mathrm{Co}$ et le ${ }^{54} \mathrm{Mn}$, ont été détectés dans moins de $3 \%$ des bioindicateurs terrestres (lichens et bryophytes), prélevés pour la plupart à proximité des sites marins (installation de Flamanville en particulier) à des niveaux d'activités inférieurs à $10 \mathrm{~Bq} \mathrm{~kg}^{-1} \mathrm{sec}$. Ces résultats peuvent être en partie attribués à des apports par les embruns marins eux-mêmes porteurs d'une contamination liée aux rejets liquides effectués en mer non seulement par les CNPE eux-mêmes, mais aussi par les installations de COGEMA-La Hague (Fraizier et al. 1977). La présence de ces radionucléides a également été révélée dans certains échantillons d'eau d'irrigation, et, à des niveaux proches des limites de détection, dans les sols et végétaux irrigués. Les cas de marquage de l'environnement terrestre pas les apports liés aux rejets atmosphériques des CNPE mis en évidence lors des études radioécologiques annuelles sont rares et restent ponctuels.

Seul le ${ }^{137} \mathrm{Cs}$ est présent dans la majorité des échantillons de l'environnement terrestre analysés (et notamment dans tous les échantillons de sol). Ce radionucléide, hérité des retombées de l'accident de Tchernobyl et des essais d'armes nucléaires, constitue le contributeur principal à l'activité des émetteurs gamma artificiels. La figure 5 permet d'illustrer la forte variabilité qui existe entre les résultats de mesures de ${ }^{137} \mathrm{Cs}$ pour une même matrice analysée, ici les sols cultivés. Outre la variabilité naturelle (liée en particulier aux différences de nature des sols), il existe des différences entre les zones géographiques où ont été réalisés 


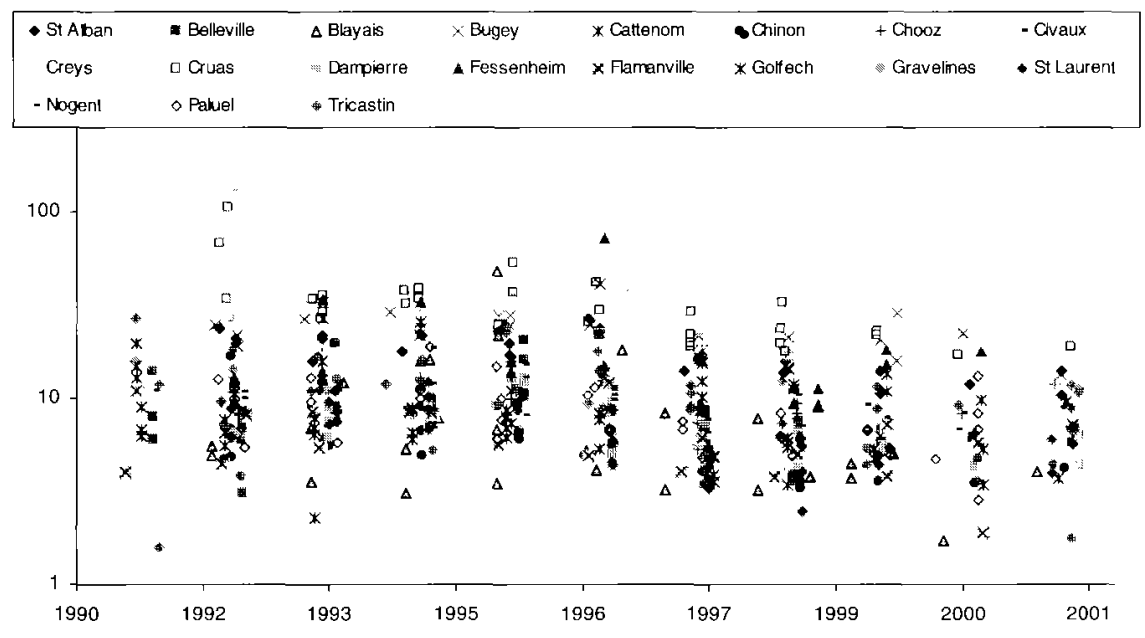

Figure 5 - Activités massiques de ${ }^{137}$ Cs mesurées dans les sols cultivés autour de chaque CNPE étudié entre 1991 et $2001\left(\mathrm{~Bq}^{-1} \mathrm{sec}\right)$.

${ }^{137}$ Cs mass activities measured in cultivated soils around each NPP studied between 1991 and $2001\left(B q \mathrm{~kg}^{-1} \mathrm{dry}\right)$.

les prélèvements. Ainsi, les sites de la vallée du Rhône et de l'est de la France montrent des niveaux de contamination en ${ }^{137} \mathrm{Cs}$ supérieurs aux sites situés à l'ouest de la France, rendant compte des dépôts plus importants qui se sont produits suite à l'accident de Tchernobyl.

Les activités en ${ }^{134} \mathrm{Cs}$, mesurables dans seulement $13 \%$ des échantillons de sols, sont beaucoup plus faibles (moins de $2 \mathrm{~Bq} \mathrm{~kg}^{-1}$ dans les sols cultivés). Ce radionucléide n'a quasiment jamais été quantifié significativement dans les végétaux, excepté dans le cas particulier des bryophytes où l'on dispose de valeurs significatives de ${ }^{134} \mathrm{Cs}$ pour près de $40 \%$ des échantillons analysés. Depuis 1998 , les activités mesurées dans les échantillons de l'environnement terrestre pour cet isotope, dont la période est relativement courte, sont, dans la plupart des cas, inférieures aux limites de détection.

\subsubsection{Milieu aquatique continental}

Dans les fleuves recevant les rejets liquides de CNPE, les taux de détection des radionucléides artificiels traceurs des ces apports sont plus importants que dans le milieu terrestre (Tab. I). La détection de ${ }^{60} \mathrm{Co}, \mathrm{d}^{110 \mathrm{~m}} \mathrm{Ag}$ ou de ${ }^{54} \mathrm{Mn}$ à l'amont des fleuves, hors de l'influence des CNPE, reste exceptionnelle (respectivement 3, 2 et 


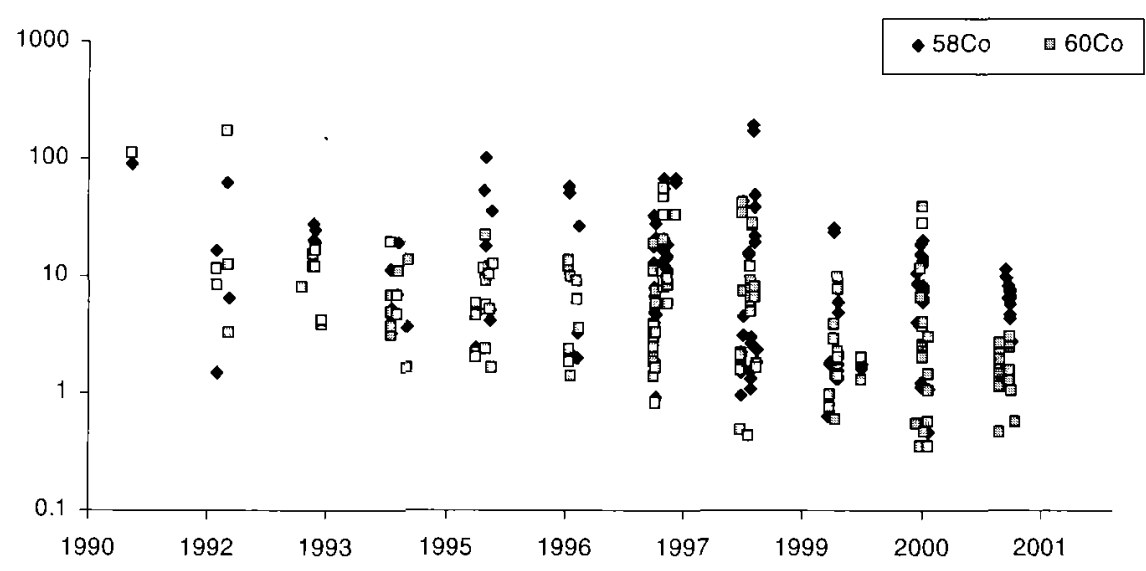

Figure 6-Activités en ${ }^{60} \mathrm{Co}$ et ${ }^{58}$ Co mesurées dans les bryophytes en aval des CNPE (Bq $\mathrm{kg}^{-1}$ sec). ${ }^{60} \mathrm{Co}$ and ${ }^{58} \mathrm{Co}$ activities measured in aquatic mosses downstream NPPs (Bq $\mathrm{kg}^{-I}$ dry).

1 fois en onze ans), et est probablement liée à des rejets de centres industriels ou de recherche. En revanche, en aval des CNPE, le ${ }^{60}$ Co est décelé dans $48 \%$ des échantillons de sédiments et $39 \%$ des échantillons végétaux prélevés, matrices présentant les plus fortes activités massiques, d'ailleurs souvent supérieures à celles du ${ }^{137} \mathrm{Cs}$. Les activités mesurées fluctuent en fonction des périodes et des lieux de prélèvement, mais ont tendance à diminuer globalement depuis 1991 (Fig. 6). Le ${ }^{60} \mathrm{Co}$ est mesurable dans moins de $5 \%$ des échantillons de poissons, à des niveaux d'activité très faibles ( $95 \%$ des valeurs sont inférieures à $1,3 \mathrm{~Bq} \mathrm{~kg}^{-1} \mathrm{sec}$ ).

Le ${ }^{58}$ Co est mesurable dans $31 \%$ des échantillons végétaux prélevés à l'aval des sites. Ses activités massiques sont proches de celles du ${ }^{60} \mathrm{Co}$ et suivent la même évolution temporelle (Fig. 6).

$\mathrm{L}^{110 \mathrm{~m}} \mathrm{Ag}$ et le ${ }^{54} \mathrm{Mn}$ sont détectés dans moins de $20 \%$ des prélèvements effectués. $\mathrm{L}^{110 \mathrm{~m}} \mathrm{Ag}$ est majoritairement associé à la fraction particulaire (sédimentée et piégée dans les bryophytes) alors que le ${ }^{54} \mathrm{Mn}$ est presque exclusivement détecté dans les végétaux. Les valeurs d'activités pour ces deux radionucléides sont généralement très faibles, quelques valeurs proches de $10 \mathrm{~Bq} \mathrm{~kg}^{-1} \mathrm{sec}$ ayant toutefois été mesurées épisodiquement à proximité de chacun des sites.

$\mathrm{L}^{131}{ }^{131}$ est détecté dans plus de $50 \%$ des végétaux dans lesquels il est recherché, à l'amont comme à l'aval des installations. Les activités les plus fortes sont mesurées dans les phanérogames, allant parfois jusqu'à plusieurs centaines de becquerels par kilogramme de matière sèche. Entre 1991 et 2001, les activités 
massiques de ce radionucléide sont restées globalement du même ordre de grandeur, ne mettant pas en évidence d'apport par les CNPE.

Le ${ }^{124} \mathrm{Sb}$ a été détecté moins de 10 fois en 11 ans en aval des sites.

Enfin, les activités en ${ }^{137} \mathrm{Cs}$ sont supérieures à la limite de détection pour plus de $84 \%$ des échantillons analysés. Observé dans $100 \%$ des échantillons de sédiments depuis 1991, c'est dans cette matrice que son activité massique est la plus importante. De manière générale, les niveaux d'activités en ${ }^{137} \mathrm{Cs}$ mesurés dans l'environnement aquatique en amont de tout site nucléaire (de 1 à $35 \mathrm{~Bq} \mathrm{~kg}^{-1} \mathrm{sec}$ entre 1991 et 2001) décroissent réguljèrement depuis 1991, avec une période moyenne effective inférieure à sa période radioactive $\left(\mathrm{T}_{1 / 2}=30,2\right.$ ans $)$.

\subsubsection{Milieu marin}

Outre le ${ }^{137} \mathrm{Cs}$, les radionucléides artificiels le plus souvent mesurés de manière significative dans le milieu marin sont $1 e^{60} \mathrm{Co}$ et $\mathrm{l}^{110 \mathrm{~m}} \mathrm{Ag}$. Le tableau I présente, pour ces trois radionucléides, les gammes d'activités mesurées significativement dans les différents échantillons du milieu marin prélevés lors des études radioécologiques des sites de Flamanville, Paluel, Gravelines et Penly.

Le ${ }^{60}$ Co est détecté dans plus de $80 \%$ des algues, des mollusques et des sédiments. Les activités les plus élevées (de l'ordre de 15 à $20 \mathrm{~Bq} \mathrm{~kg}^{-1}$ sec) sont mesurées dans les sédiments vaseux des ports et des amenées d'eau des centrales. Le ${ }^{60} \mathrm{Co}$ est, avec le ${ }^{137} \mathrm{Cs}$, le seul radionucléide artificiel émetteur gamma présentant des activités supérieures aux limites de détection dans plus de $3 \%$ des échantillons de poissons. $\mathrm{L}^{, 110 \mathrm{~m}} \mathrm{Ag}$ est détecté dans plus d'un échantillon sur trois. Il est notamment observé dans l'environnement proche des rejets, en particulier dans les algues et les mollusques. Les stations témoins, situées à plus de $10 \mathrm{~km}$ des sites, ne présentent aucun marquage significatif dû à ce radionucléide. Les niveaux les plus élevés sont mesurés dans les algues du canal de rejet du CNPE de Gravelines ( 10 à $50 \mathrm{~Bq} \mathrm{~kg}{ }^{-1} \mathrm{sec}$ ). Le ${ }^{58} \mathrm{Co}$ est détecté dans environ $5 \%$ de l'ensemble des échantillons. Le ${ }^{125} \mathrm{Sb}$, le ${ }^{54} \mathrm{Mn}$ et le ${ }^{134} \mathrm{Cs}$ sont détectés moins fréquemment.

Le ${ }^{106} \mathrm{Ru}$, qui n'est détecté que dans le milieu marin, est mesuré significativement dans environ $10 \%$ des échantillons. Au cours de ces onze années, les valeurs maximales de ${ }^{106} \mathrm{Ru}\left(10\right.$ à $20 \mathrm{~Bq} \mathrm{~kg}^{-1} \mathrm{sec}$ ) sont observées dans les mollusques prélevés à proximité de Flamanville, CNPE proche de l'usine de retraitement des combustibles usés de COGEMA-La Hague.

Globalement la fréquence de détection des radionucléides émetteurs gamma artificiels dans le milieu marin et leurs niveaux d'activité ont diminué au cours de la décennie. 


\section{Discussion}

La partie précédente a permis d'énoncer un bilan des principaux résultats acquis au cours de 11 années d'études de l'environnement des sites nucléaires de production d'électricité. Ici, l'objectif est d'utiliser les connaissances que l'on a des différentes sources de radioactivité artificielle afin d'analyser ces résultats en terme de marquage de l'environnement attribuable au fonctionnement des CNPE.

De manière générale, outre les isotopes du césium, hérités des retombées globales, les radionucléides traceurs des rejets des CNPE n'ont été que rarement détectés dans le milieu terrestre. Quand tel a été le cas, l'origine de la contamination a souvent pu être identifiée, et n'est, dans la majorité des cas, pas liée aux rejets atmosphériques des sites de production.

Dans les milieux aquatiques continental et marin, ce marquage est mis en évidence depuis 1991. Toutefois, dans le milieu marin, et en particulier à proximité du site de Flamanville, il est souvent difficile à mettre en évidence du fait de l'influence des rejets de l'usine de COGEMA-La Hague. Le choix des radionucléides traceurs des différents termes sources est alors primordial. Certains radionucléides émetteurs gamma, comme le ${ }^{106} \mathrm{Ru}$, sont caractéristiques des rejets de l'usine de COGEMA-La Hague. D'autres, comme le ${ }^{60} \mathrm{Co}$, sont communs aux rejets liés au fonctionnement normal des CNPE et de l'usine de retraitement. Cependant, la quantité de ce radionucléide rejeté par l'usine COGEMA-La Hague est deux à trois ordres de grandeur plus élevée que celle rejetées par les CNPE (Fig. 7). L'ensemble des résultats de mesures du ${ }^{60} \mathrm{Co}$ acquis sur les échantillons d'algues, de mollusques, de crustacés et de sédiments sur l'ensemble des côtes de la Manche montrent que la contamination du milieu dépend de la situation géographique par rapport à la zone de rejets des effluents liquides de COGEMA-La Hague. Ainsi, l'environnement de la Centrale de Flamanville, plus proche du Cap de La Hague, est caractérisée par un marquage plus important en ${ }^{60} \mathrm{Co}$ (Fig. 7). $\mathrm{L}^{, 110 \mathrm{~m}} \mathrm{Ag}$ et le ${ }^{58} \mathrm{Co}$, plus épisodiquement le ${ }^{54} \mathrm{Mn}$, sont davantage représentatifs de la signature des CNPE, et ne sont détectables que dans leur environnement proche.

Il est difficile de déceler un éventuel marquage attribuable aux rejets des CNPE à partir de l'analyse des niveaux d'activité des isotopes 137 et $134 \mathrm{du}$ césium. Le calcul des rapports d'activités ${ }^{137} \mathrm{Cs} /{ }^{134} \mathrm{Cs}$, quand cela est possible, constitue un élément d'expertise complémentaire et permet de rendre compte d'un apport local. Les figures 8 et 9 représentent respectivement les rapports ${ }^{137} \mathrm{Cs} /{ }^{134} \mathrm{Cs}$ obtenus sur les échantillons du milieu terrestre et ceux du milieu aquatique continental (toutes matrices confondues). Après 1998 , le ${ }^{134} \mathrm{Cs}$, dont l'activité issue des retombées de Tchernobyl a fortement décru, n'est plus quantifié significativement que dans quelques cas. 

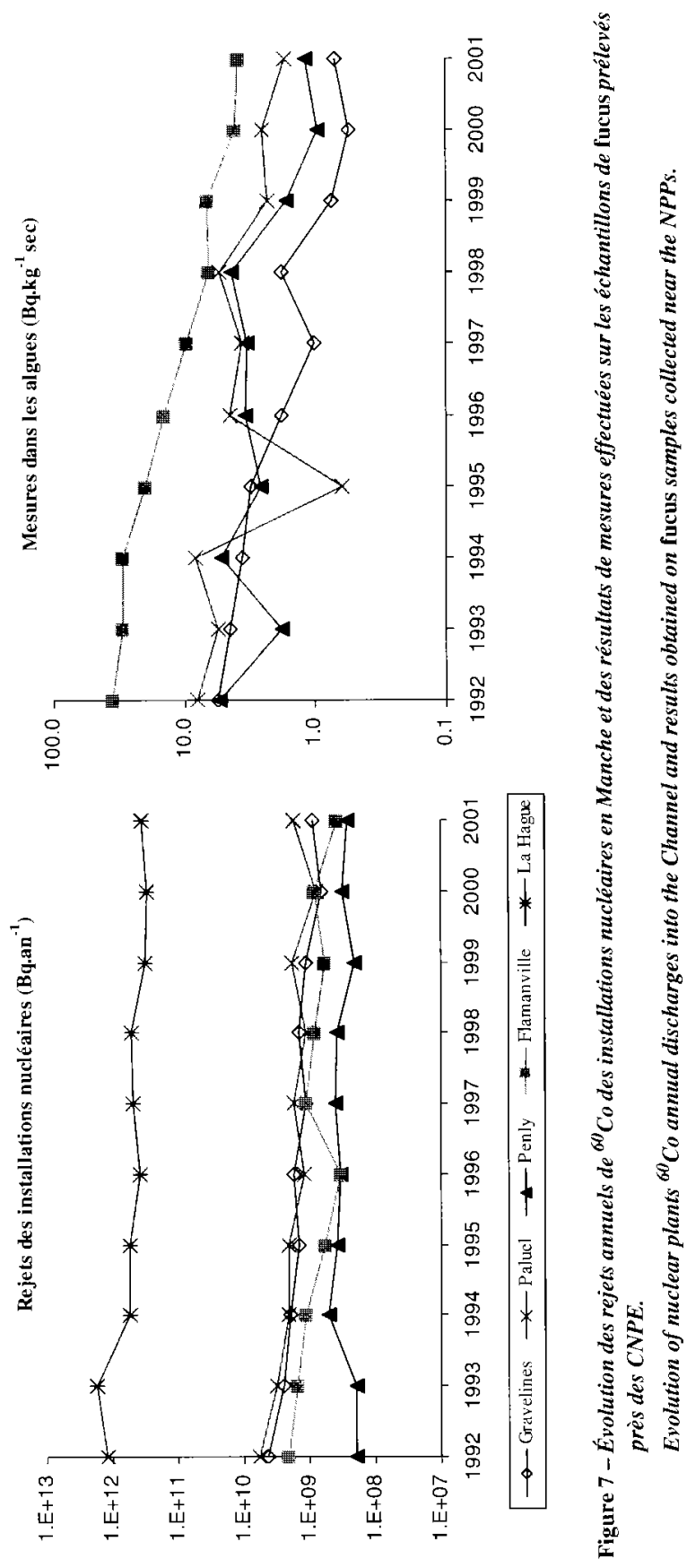


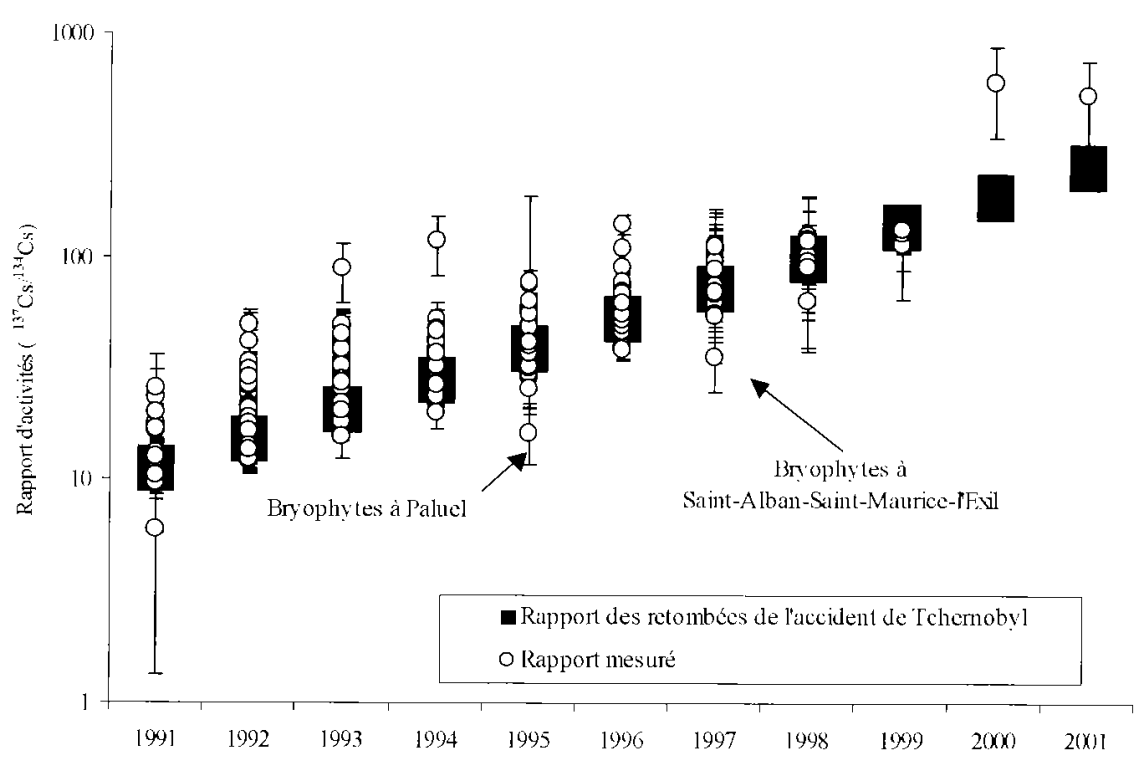

Figure 8-Rapports d'activités $\left({ }^{137} \mathrm{Cs} /{ }^{134} \mathrm{Cs}\right)$ mesurés dans l'environnement terrestre et rapports caractéristiques des retombées de l'accident de Tchernobyl.

${ }^{137} \mathrm{Cs} /{ }^{134} \mathrm{Cs}$ activity ratios in terrestrial environment and Chernobyl fallout characteristic ratios.

Dans le milieu terrestre (Fig. 8), la majorité des résultats se situent dans une gamme de valeurs supérieures ou égales aux rapports caractéristiques des retombées de l'accident de Tchernobyl. Quelques résultats se situent en deçà de ces valeurs. En prenant en compte les incertitudes de mesures, seuls deux échantillons de mousses terrestres présentent des valeurs de rapports ${ }^{137} \mathrm{Cs} /{ }^{134} \mathrm{Cs}$ significativement inférieures. Ces bryophytes ont été prélevées à proximité des sites de Paluel en 1995 et de Saint-Alban en 1997.

Par contre, les résultats obtenus sur les échantillons collectés dans les cours d'eau mettent clairement en évidence un marquage systématique par les effluents liquides rejetés par les sites électronucléaires (Fig. 9). Le césium provenant du drainage des bassins versants des cours d'eau ne constitue plus le contributeur unique aux activités mesurées en aval des CNPE. Les rapports d'activités calculés, qui sont dans la plupart des cas inférieurs au rapport caractéristique des retombées de l'accident de Tchernobyl, témoignent en effet d'un mélange avec une source additionnelle de césium dans les cours d'eau. 


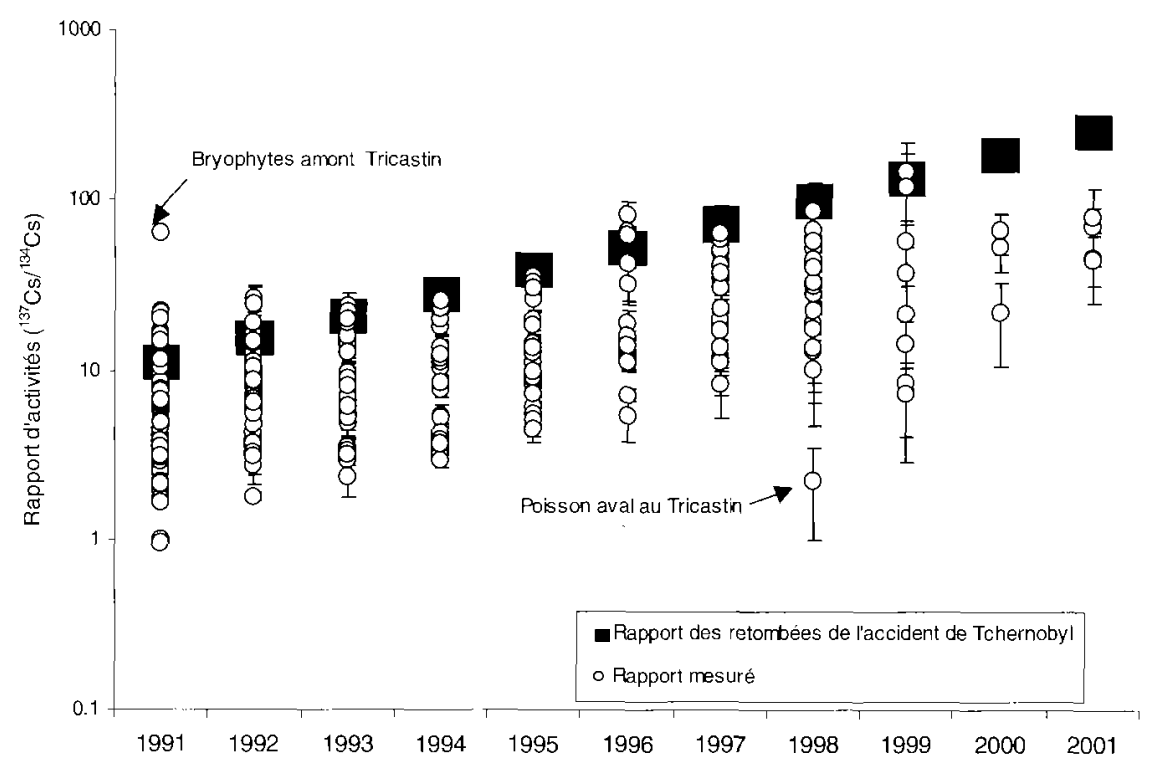

Figure 9-Rapports d'activités $\left({ }^{137} \mathrm{Cs} /{ }^{134} \mathrm{Cs}\right)$ mesurés dans les matrices aquatiques et rapports caractéristiques des retombées de l'accident de Tchernobyl.

${ }^{137} \mathrm{Cs} /{ }^{134} \mathrm{Cs}$ activity ratios in aquatic environment and Chernobyl fallout characteristic ratios.

Signalons que la présence $\mathrm{d}^{131} \mathrm{I}$, de courte période $\left(\mathrm{T}_{1 / 2}=8\right.$ jours) dans les cours d'eau français est due principalement aux rejets des centres hospitaliers. Les rejets effectués par voie liquide par les CNPE n'ont pas été mis en évidence lors des suivis radioécologiques annuels réalisés entre 1991 et 2001.

\section{Conclusion}

Les études radioécologiques menées annuellement entre 1991 et 2001 autour des installations nucléaires de production d'électricité françaises ont conduit à l'analyse par spectrométrie gamma de plus de 5000 échantillons de l'environnement, issus des milieux terrestre, aquatique continental et marin. Le choix des stratégies de prélèvements, dont la pertinence doit rester une des préoccupations essentielles de ce type d'études, vise à répondre à trois principaux objectifs : établir la présence ou l'absence de marquage de l'environnement par les rejets industriels, quantifier les niveaux d'activités des différents radionucléides pour chaque type de matrice, établir des comparaisons entre les différents sites étudiés. La connaissance à la fois quantitative et qualitative des différents termes sources est alors un point essentiel, qui permet l'interprétation des résultats obtenus. 
Les séries de résultats acquis en 11 ans permettent de présenter des gammes de valeurs représentatives des teneurs en radionucléides naturels émetteurs gamma dans les différents compartiments de l'environnement. Ces bilans statistiques sont cohérents avec les valeurs de référence citées dans la littérature, et attestent de la qualité des résultats sur lesquels reposent l'expertise.

Les rejets atmosphériques chroniques de radionucléides artificiels émetteurs gamma des CNPE ne donnent lieu à aucun marquage quantifiable de l'environnement. Dans le milieu terrestre, la présence de radioactivité artificielle est principalement liée à la rémanence des isotopes 137 et dans une moindre mesure 134 du césium, héritée des retombées atmosphériques de Tchernobyl et des essais d'armes nucléaires. En revanche, les rejets d'effluents liquides dans les cours d'eau ou en mer, qui concernent des activités plus importantes, sont mis en évidence dans plusieurs matrices prélevées en aval des points de rejets. ${ }^{60} \mathrm{Co},{ }^{58} \mathrm{Co}$, ${ }^{110 \mathrm{~m}} \mathrm{Ag}$ et ${ }^{54} \mathrm{Mn}$ sont les principaux traceurs de ces apports. De plus, la détermination des rapports d'activités ${ }^{137} \mathrm{Cs} /{ }^{134} \mathrm{Cs}$ est également un bon moyen de mettre en évidence un marquage du milieu par les rejets des CNPE. Les végétaux aquatiques sont les meilleurs marqueurs des évolutions des flux de radionucléides dans ce milieu, et témoignent notamment des diminutions des rejets entre 1991 et 2001. Les rejets liquides des sites de production situés sur le littoral français (Flamanville, Penly, Paluel et Gravelines) conduisent également à un marquage décelable à proximité des zones de rejets. Cependant, les rejets dans la Manche de plusieurs radionucléides émetteurs gamma par les installations nucléaires de COGEMA-La Hague peuvent s'ajouter aux apports liés aux CNPE. Les activités mesurées dans les compartiments marins ne sont alors pas directement dépendantes et corrélées avec les activités rejetées par les centrales à l'instar des résultats obtenus en Suède (Wallberg et Moberg, 2002).

Outre leur intérêt pour l'analyse du marquage radioécologique attribuable aux CNPE français, les séries de résultats acquis annuellement depuis 1991 constituent une source importante de données, utiles à la compréhension des processus de distribution de la radioactivité dans l'environnement, et sur lesquelles les différents travaux menés à l'IRSN peuvent s'appuyer.

Remerciements. Les auteurs remercient Électricité de France, qui leur a permis de mener à bien les études dont les résultats sont synthétisés ici. Merci également à COGEMA-La Hague dont les données fournies sont essentielles à l'analyse des résultats de mesures obtenus en Manche. Ce document s'appuie sur le travail réalisé annuellement depuis 1991 par plusieurs techniciens et ingénieurs qui doivent être également remerciés pour leur contribution. 


\section{RÉFÉRENCES}

Bouisset P., Calmet D. (1997) Hyper pure gamma-ray spectrometry applied to low-level environmental sample measurements, in: Proceedings of the International Workshop on status of Measurement Techniques for the Identification of Nuclear Signature, Geel, February 25-27, 1997.

EDF (1995) Nucléaire et Environnement, Édition 1995.

EDF (2001) Nucléaire et Environnement, Rapport annuel.

Foulquier L., Bretheau F. (1998) Les installations nucléaires et l'environnement, Méthode d'évaluation de l'impact radioécologique et dosimétrique, Coll. IPSN. EDP Sciences, Les Ulis, 172 p.

Fraizier A., Masson M., Guary J.C. (1977) Recherches préliminaires sur le rôle des aérosols dans le transfert de certains radiófléments du milieu marin au milieu terrestre, J. Rech. Atmos. 11(1), 49-60.

Hölgye Z., Filgas R. (1995) Inventory of ${ }^{238} \mathrm{Pu}$ and ${ }^{239+240} \mathrm{Pu}$ in the soil of Czechoslovakia in 1990 , J. Environm. Radioact. 27, 181-189.

IRSN (1992 à 2002) Suivi radioécologique annuel des centrales nucléaires françaises (spectrométrie $\gamma$ ) - Rapports annuels.

Mitchell P.I., Sanchez-Cabeza J.A., Ryan T.P., Mcgarry A.T., Vidal-Quatras A. (1990) Preliminary estimates of cumulative caesium and plutonium deposition in the Irish terrestrial environment, J. Radioanal. Nucl. Chem. 138, 241-256.

Ramade F. (1998) Dictionnaire encyclopédique des sciences de l'eau. Édiscience international, $786 \mathrm{p}$.

Renaud P., Beaugelin K., Maubert H., Ledenvic P. (1999) Les retombées en France de l'accident de Tchernobyl, Conséquences radioécologiques et dosimétriques, Coll. IPSN. EDP Sciences, Les Ulis, $146 \mathrm{p}$.

Renaud P., Pourcelot L., Métivier J.M., Morello M. (2003) Mapping of ${ }^{137}$ Cs deposition over eastern France 16 years after the Chernobyl accident, Sci. Tot. Environm. 309, 257-264.

UNSCEAR (1988) Sources, effects and risks of ionising radiation, Report to the General Assembly, Vol. I: Sources.

UNSCEAR (2000) Sources and effects of ionising radiation, Report to the General Assembly, Vol. I: Sources.

Wallberg P., Moberg L. (2002) Evaluation of 20 years of environmental monitoring data around Swedish nuclear installations, J. Environm. Radioact. 63, 117-133. 